\title{
NEUROIMAGING AND LOCALIZATION
}

\author{
Hermann Stefan \\ University Hospital Erlangen, Department of Neurology
}

\begin{abstract}
For classification of epilepsies and presurgical evaluation of patients with pharmacoresistent epilepsies in addition to case history and neurological examination neuroimaging became increasingly important. Advanced neuroimaging in addition to structural MRI and functional MRI concerns studies of cerebral metabolism like FDG-PET, FET-PET, perfusion analysis during epileptic seizures as ictal SPECT.

High resolution recordings of neuronal epileptic activity can be obtained by means of electrophysiology using EEG or magnetoencephalography (MEG).
\end{abstract}

MEG is a contactless multichannel recording technique, which is less influenced by the different conductivities of the tissues in the head (skin, skull, brain parenchyma, cerebrospinal fluid).

Multimodal imaging combines the results of the different techniques for optimized information of individual anatomy, structural and functional changes. By this way noninvasive investigations permit the visualization of epileptogenic lesions and functional cortex. This information can be displayed in the operating room.

Examples will be demonstrated.
Prof. Dr. Hermann Stefan

University Hospital Erlangen,

Department of Neurology - Biomagntism,

Schwabachanlage 10, 91054 Erlangen/ Germany,

Phone: +49 9131 8536989, Fax: +49 91318534226 ,

Email: hermann.stefan@uk-erlangen.de 